\title{
Respondent driven sampling of wheelchair users: A lack of
}

\section{traction? [version 1; peer review: 3 approved with}

\section{reservations]}

John A. Bourke1,2, Philip J. Schluter1,3, E. Jean C. Hay-Smith4, Deborah L. Snell2,5

${ }^{1}$ School of Health Sciences, University of Canterbury, Canterbury, 8140, New Zealand

${ }^{2}$ Burwood Academy of Independent Living, Christchurch, 8140, New Zealand

${ }^{3}$ School of Nursing, Midwifery and Social Work, The University of Queensland, Brisbane, 4072, Australia

${ }^{4}$ Rehabilitation Teaching and Research Unit, University of Otago Wellington, Wellington, 6242, New Zealand

${ }^{5}$ Department of Orthopaedic Surgery and Musculoskeletal Medicine, University of Otago Christchurch, Christchurch, 8140, New Zealand

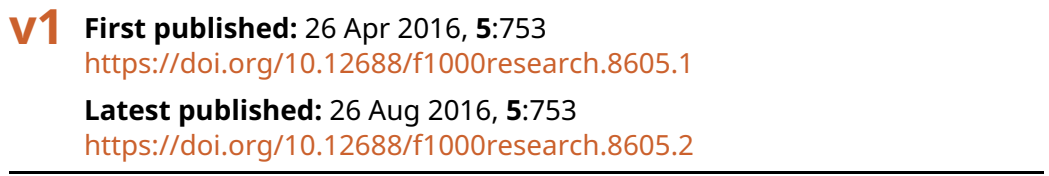

\section{Abstract}

Background: Internationally wheelchair users are an emerging demographic phenomenon due to their rapidly increasing life-span coupled with accelerated general population ageing. While having significant healthcare and social implications, basic robust epidemiological information of wheelchair users is often lacking due in part to this population's "hidden" nature. Increasingly popular in epidemiological research, Respondent Driven Sampling (RDS) provides a mechanism for generating unbiased population-based estimates for hard-to-reach populations, overcoming biases inherent within other sampling methods. This paper reports the first published study to employ RDS amongst wheelchair users.

Methods: Between October 2015 and January 2016, a short, successfully piloted, internet-based national survey was initiated. Twenty seeds from diverse organisations were invited to complete the survey then circulate it to peers within their networks following a welldefined protocol. A predetermined reminder protocol was triggered when seeds or their peers failed to respond. All participants were entered into a draw for an iPad.

Results: Overall, 19 people participated ( 9 women); 12 initial seeds, followed by seven second-wave participants arising from four seeds. Completion time for the survey ranged between 7 and 36 minutes. Despite repeated reminders, no further people were recruited.

Discussion: While New Zealand wheelchair user numbers are unknown, an estimated $14 \%$ of people have physical impairments that limited mobility. The 19 respondents generated from adopting the

\section{Open Peer Review}

\begin{tabular}{lccc} 
Approval Status & 1 & 2 \\
\hline version 2 & & & \\
(revision) & view & view & view \\
26 Aug 2016 & $?$ & $?$ & $?$ \\
version 1 & $?$ & $?$ & view \\
26Apr 2016 & view & view &
\end{tabular}

1. Jesse Kokaua, University of Otago, Dunein, New Zealand

2. John F. Smith, Kohn Kaen University, Khon Kaen, Thailand

3. A James O'Malley, Geisel School of Medicine at Dartmouth, Lebanon, USA

Any reports and responses or comments on the article can be found at the end of the article. 
RDS methodology here thus represents a negligible fraction of wheelchair users in New Zealand, and an insufficient number to ensure equilibrium. While successful in other hard-to-reach populations, applying RDS methodology to wheelchairs users requires further consideration. Formative research exploring areas of network characteristics, acceptability of RDS, appropriate incentive options, and seed selection amongst wheelchair users is needed.

\section{Keywords}

Wheelchair users, Disability, Respondent driven sampling , Social epidemiology, Sampling approaches

Corresponding author: John A. Bourke (john.bourke@pg.canterbury.ac.nz)

Competing interests: No competing interests were disclosed.

Grant information: This paper reports on a section of JAB's doctoral research, which was supported by a University of Canterbury Doctoral Scholarship and a research scholarship from the Burwood Academy of Independent Living.

The funders had no role in study design, data collection and analysis, decision to publish, or preparation of the manuscript.

Copyright: @ 2016 Bourke JA et al. This is an open access article distributed under the terms of the Creative Commons Attribution License, which permits unrestricted use, distribution, and reproduction in any medium, provided the original work is properly cited.

How to cite this article: Bourke JA, Schluter PJ, Hay-Smith EJC and Snell DL. Respondent driven sampling of wheelchair users: A lack of traction? [version 1; peer review: 3 approved with reservations] F1000Research 2016, 5:753 https://doi.org/10.12688/f1000research.8605.1

First published: 26 Apr 2016, 5:753 https://doi.org/10.12688/f1000research.8605.1 


\section{Introduction}

Robust epidemiological research generally requires data collection from representative samples of the population of interest, and effective modes of sampling contact are essential ${ }^{1}$. Such effective modes can be difficult in hard-to-reach populations where no (or inadequate) sampling frames exist. Traditional chain-referral sampling approaches are inherently biased in their participant selection methods; a bias that is compounded as recruitment waves continue. Respondent Driven Sampling (RDS) was developed to counter these biases, employing specific data collection and statistical analysis methods which enable valid populationbased estimates ${ }^{2-4}$. Despite wider adoption of RDS, and its successful application in many topic areas, methodological concerns have been raised. RDS estimates are, at times, more variable than expected $^{5}$, and some sampling patterns appear to violate core RDS assumptions $s^{6,7}$.

The prevalence of wheelchair users has rapidly increased over the last half century due in part to advancing medical care, ageing populations, increasing community supports, increased prescription of wheelchairs, and changes in attitudes to disablement such that people may feel less stigmatised about using a wheelchair ${ }^{8,9}$. Despite this, robust epidemiological research in this group is scant ${ }^{10}$. Contacting wheelchair users in the community is challenging. Recruitment approaches are often limited to using disability organisations and personal contacts, which can differentially exclude many wheelchair users ${ }^{11}$. Consequently, wheelchair users frequently constitute a 'hidden population', under-researched and excluded from population estimates ${ }^{12}$. Furthermore, many countries, including New Zealand, have yet to establish registries of wheelchair users which could provide a sampling frame ${ }^{13}$.

Here we report our experience of applying a RDS methodology to a survey of wheelchair users in New Zealand. To our knowledge this is the first time RDS has been applied to people who use wheelchairs, and could potentially offer a significant new sampling approach in epidemiology and disability fields.

\section{Methods}

Open from October 2015 until January 2016, this study employed a short internet-based national survey. Administered through the Surveymonkey ${ }^{\mathrm{TM}}$ website, an information sheet and video were embedded within the survey preamble (see Supplementary material). The information sheet stated that informed consent was implied through the voluntary participation in the survey. Ethical approval was obtained from the University of Canterbury Human Ethics Committee (reference HEC 2015/117). Eligibility criteria included: wheelchair use as the primary form of mobility; being a New Zealand resident; aged 16 years or more; being able to read English; having internet access; and, having an operational email account.

Invitations seeking 'seed' participants were circulated to various national disability organisations serving members with a range of impairments that lead to wheelchair use, including the New Zealand Spinal Trust, the Earthquake Disability Leadership Group, the Multiple Sclerosis Society of New Zealand, the Cerebral Palsy Society of New Zealand, and CCS Disability Action.
People expressing interest in being seeds contacted the researcher, who confirmed eligibility and then sent a recruitment code and a link to the survey website. Once a participant completed the survey, they were thanked and emailed three unique recruitment codes. Participants were asked to email one code and the survey link to three other persons they knew who were likely to satisfy the eligibility criteria. This process was envisaged to continue for multiple recruitment waves. Participation was incentivised (an entry into a draw to win an iPad); one entry for completing the survey, and another when each person they recruited completed the survey. Recruitment chains were tracked through tracing the recruitment codes. A predetermined reminder protocol was triggered when seeds or their peers failed to respond.

\section{Results}

Twelve seed participants completed the survey. A further seven participants were recruited from the second wave, from a total of four seeds, and none were recruited from the third wave. The final sample size $(n=19)$ failed to satisfy the statistical requirements needed to reach equilibrium, the point at which the sample composition becomes independent of the initial seeds, thereby enabling the calculation of unbiased population estimates ${ }^{4}$. Mean age of participants was 55.6 years (range: 28-73 years), and nine were women. Eighteen were identified as New Zealand European and one was identified as New Zealand Māori. Reasons for using a wheelchair included impaired mobility resulting from spinal cord injury $(n=10)$, cerebral palsy $(n=2)$, spina bifida $(n=2)$, muscular dystrophy $(n=2)$, poliomyelitis $(n=2)$, and arthritis $(n=1)$. Survey completion time ranged between 7 and 36 minutes.

\section{Discussion}

Despite a rigorous recruitment process and offering incentivising participation, our use of RDS failed as an effective sampling approach amongst wheelchair users in New Zealand. There are a number of possible explanations as to why this occurred. The target population of the study was novel compared with hidden populations generally targeted by RDS studies. Research using RDS typically samples stigmatised populations, such as those with greater risk of HIV, including injecting drug users ${ }^{6}$, men who have sex with men ${ }^{7,14}$, and sex workers ${ }^{15}$. Wheelchair users have experienced increased integration into many societies in recent years and are arguably less stigmatised when compared to populations traditionally sampled using RDS. Although the precise mechanism by which perceived stigma might affect RDS participation is unknown it, nonetheless, remains noteworthy. Second, the use of an unguaranteed reward (entry into a draw for an iPad) for survey completion has not been previously reported in RDS studies. This lack of guaranteed reward may have influenced participation. In addition, RDS studies often offer participants additional non-monetary free services related to the mitigation of HIV risk, such as counselling and educational material ${ }^{16}$.

Until such time as these factors, and their implications for recruitment, are better understood we feel that using RDS for recruiting wheelchairs users may have limited merit, and recommend formative research to optimise success. Exploring the areas of network characteristics, acceptability of RDS, appropriate incentive options, and seed selection have all been suggested as 
important for assessing the feasibility and appropriateness of RDS in certain populations ${ }^{15}$. Specifically, formative research regarding specific seed selection is warranted. Motivated seeds with large network contacts can improve recruitment effectiveness. Indeed, one RDS study exploring people who inject drugs in Sydney Australia reported that $80 \%$ of their participants resulted from one seed ${ }^{17}$.

\section{Conclusions}

Wheelchair users are an increasingly prevalent population in society who often lack an adequate sampling frame, and sampling approaches enabling valid population based estimates are becoming increasingly necessary. This paper reported the failure of RDS to survey wheelchair users. Despite the unsuccessful recruitment in this study, further research exploring the application of RDS with wheelchair users is recommended before discounting this sampling approach in this population.

\section{Data availability}

Data are available upon request from the corresponding author to protect participant identity. Demographic data will be pooled to protect participant identity, as individual-level demographic data could be theoretically traceable due to the small sample size, and suspected small national population of wheelchair users.

\section{Consent}

All participants were informed that the voluntary completion of the survey implied informed consent, including for the publication of survey data.

\section{Author contributions}

All authors contributed to the design of the study. JAB conducted the data collection and prepared the first draft of the manuscript. All authors contributed to subsequent drafts and the final manuscript.

\section{Competing interests}

No competing interests were disclosed.

\section{Grant information}

This paper reports on a section of JAB's doctoral research, which was supported by a University of Canterbury Doctoral Scholarship and a research scholarship from the Burwood Academy of Independent Living.

The funders had no role in study design, data collection and analysis, decision to publish, or preparation of the manuscript.

\section{Supplementary material}

Internet-based national survey.

Click here to access the data.

Information sheet.

Click here to access the data.

1. Sinclair M, O'Toole J, Malawaraarachchi M, et al:: Comparison of response rates and cost-effectiveness for a community-based survey: postal, internet and telephone modes with generic or personalised recruitment approaches. $B M C$ Med Res Methodol. 2012; 12: 132.

PubMed Abstract | Publisher Full Text | Free Full Text

2. Heckathorn DD: Respondent-driven sampling: A new approach to the study of hidden populations. Soc Probl. 1997; 44(2): 174-199.

Publisher Full Text

3. White RG, Hakim AJ, Salganik MJ, et al:: Strengthening the Reporting of Observational Studies in Epidemiology for respondent-driven sampling studies: "STROBE-RDS" statement. J Clin Epidemiol. 2015; 68(12): 1463-1471. PubMed Abstract | Publisher Full Text | Free Full Text

4. Volz E, Heckathorn DD: Probability based estimation theory for respondentdriven sampling. J Off Stat. 2008; 24(1): 79-97. Reference Source

5. Goel S, Salganik MJ: Assessing respondent-driven sampling. Proc Natl Acad Sci U S A. 2010; 107(15): 6743-6747.

PubMed Abstract | Publisher Full Text | Free Full Text

6. Young AM, Rudolph AE, Quillen D, et al:: Spatial, temporal and relational patterns in respondent-driven sampling: evidence from a social network study of rural drug users. J Epidemiol Community Health. 2014; 68(8): 792-798. PubMed Abstract | Publisher Full Text | Free Full Text
7. Phillips G 2nd, Kuhns LM, Garofalo R, et al:: Do recruitment patterns of young men who have sex with men (YMSM) recruited through respondent-driven sampling (RDS) violate assumptions? J Epidemiol Community Health. 2014; 68(12): 1207-1212

PubMed Abstract | Publisher Full Text | Free Full Text

8. Sapey B, Stewart J, Donaldson G: Increases in wheelchair use and perceptions of disablement. Disability \& Society. 2005; 20(5): 489-505. Publisher Full Text

9. Russell JN, Hendershot GE, LeClere F, et al.: Trends and differential use of assistive technology devices: United States, 1994. Adv Data. 1997; (292): 1-9. PubMed Abstract

10. The New Zealand Convention Coalition: The Second Report of the Independent Monitoring Mechanism on the Convention of the Rights of Persons with Disabilities: Making Disability Rights Real Whakatūturu ngā Tika Hauatanga. Wellington: The New Zealand Convention Coalition, 2014 Reference Source

11. Edwards K, McCluskey A: A survey of adult power wheelchair and scooter users. Disabil Rehabil Assist Technol. 2010; 5(6): 411-419. PubMed Abstract | Publisher Full Text

12. Smaill RP: Ageing with Spinal Cord Injury in New Zealand. Christchurch University of Otago, 2014 Reference Source 
13. Fitzgerald SG, Kelleher A, Teodorski E, et al.: The development of a nationwide registry of wheelchair users. Disabil Rehabil Assist Technol. 2007; 2(6): 358-365.

PubMed Abstract | Publisher Full Text

14. Strömdahl S, Lu X, Bengtsson L, et al.: Implementation of Web-Based Respondent Driven Sampling among Men Who Have Sex with Men in Sweden. PLoS One. 2015; 10(10): e0138599.

PubMed Abstract | Publisher Full Text | Free Full Text

15. Simic M, Johnston LG, Platt L, et al.: Exploring barriers to 'respondent driven sampling' in sex worker and drug-injecting sex worker populations in Eastern
Europe. J Urban Health. 2006; 83(6 Suppl): i6-15. PubMed Abstract | Publisher Full Text | Free Full Text

16. Malekinejad M, Johnston LG, Kendall C, et al:: Using respondent-driven sampling methodology for HIV biological and behavioral surveillance in international settings: a systematic review. AIDS Behav. 2008; 12(4 Suppl): S105-S130. PubMed Abstract | Publisher Full Text

17. Paquette DM, Bryant J, Crawford $\mathrm{S}$, et al:: Conducting a respondent-driven sampling survey with the use of existing resources in Sydney, Australia. Drug Alcohol Depend. 2011; 116(1-3): 125-131.

PubMed Abstract | Publisher Full Text 


\title{
Open Peer Review
}

\section{Current Peer Review Status: ? ? ?}

\section{Version 1}

Reviewer Report 11 July 2016

https://doi.org/10.5256/f1000research.9259.r14896

(c) 2016 O'Malley A. This is an open access peer review report distributed under the terms of the Creative Commons Attribution License, which permits unrestricted use, distribution, and reproduction in any medium, provided the original work is properly cited.

\author{
A James O'Malley \\ Department of Biomedical Data Science and The Dartmouth Institute of Health Policy and Clinical \\ Practice, Geisel School of Medicine at Dartmouth, Lebanon, NH, USA
}

Title and Abstract: The title is catchy and appropriate given the content of the article.

Article content: The article is very well written and is easy to read and follow. The article would be much improved if it included an introduction to respondent drive sampling (RDS) with particular emphasis on the assumptions required for RDS to work (reaching equilibria and otherwise) written in clear terms to a lay audience. Describe what it means for the sampling process to be in equilibrium. Currently, readers do not have the necessary information to reach an informed conclusion as to why RDS did not work well in this instance or to assess whether it is applicable to their own work.

The rationale for RDS assumes the existence of a social network in which the probability of a tie between a wheelchair user and a randomly selected wheelchair user exceeds the probability of a tie between a wheelchair user and a nonuser wheelchair user. What evidence that this is true? Do wheelchair users have sufficient social and other relationships importantly enough for them to be able to name sufficient other wheelchair users for the next round of sampling. Is it possible that many wheelchair users are isolates (in the network sense) because they are highly functional in everyday life and thus have reduced need for a community of wheelchair users? I don't know the answers to these questions and still don't as the needed background information is not provided in the article.

It would have been helpful to have been told the current state of knowledge about social networks among wheelchair users along with a connection of such knowledge to the assumptions under which RDS can yield unbiased population-level inferences. I would like to see some discussion of how RDS handles scenarios such as isolates in the wheelchair user network. Presumably, the only way such individuals make it into the sample is if they were randomly selected in the first (i.e., seed) wave.

Please add some general intuition for how RDS yields unbiased population-level estimates. Discuss 
the role of and derivation of individuals' sampling probabilities and thus sampling weights for computing population estimates. For example, the work of Thompson (2006a, 2006b, 2003, 2000 see below - the 2012 text on Sampling and recent arXiv contributions) should be reviewed and used to inform the article.

References:

1. Thompson, Steven K. Targeted random walk designs. Survey Methodology 2006. 32:11-24

2. Thompson, Steven K. Adaptive web sampling. Biometrics 2006. 62:1224-1234

3. Chow, Mosuk, Thompson, Steven K. Estimation with link-tracing sampling designs - A Bayesian approach. Survey Methodology 2003. 29:197-205

4. Thompson, Steven K., Frank, Ove. Model-based estimation with link-tracing sampling designs. Survey Methodology 2000. 26:87-98

Data (if applicable): Details about the design/data for the study are lacking. It is stated that 20 wheelchair users were the initial seeds of which 12 agreed to participate (60\% response rate). It then appears as though 4 of these 12 participants culled 7 further participants in the second stage of sampling. Question: Did the remaining 8 seeds not generate any wheelchair users (i.e., does the enrolment rate for the second stage equal 7/12) or was the plan to only ask 33\% of the initial seeds for links to trace (in which case the enrolment rate is 7/4)? In the third stage, there was no addition to the sample-size. Were the 7 participants who entered at the second stage each asked to name other wheelchair users; is the enrolment rate at the third stage $0 / 7$ or $0 / x$, where $x<7$ ? Was there any limit as to how many wheelchair users a seed to subsequently enrolled person could name? A lot of these questions would be answerable if the design of the RDS was described in the article!

Conclusions: The discussion makes a number of good points that have appear sound and logical. The primary point made is that the current study may have failed because the wheelchair user population has different characteristics from populations where RDS has been used successfully. This is a valid point. But shouldn't this point have been considered when the study was design? Was there any prior information to inform the parameters of the RDS design? Maybe RDS would work quite well for the wheelchair user population as long as the number of initial seeds is much greater than 20. A more positively-framed Discussion might leave readers with a more balanced appreciation for using RDS in their own studies of wheelchair users or other new populations.

Competing Interests: No competing interests were disclosed.

\section{I confirm that I have read this submission and believe that I have an appropriate level of expertise to confirm that it is of an acceptable scientific standard, however I have significant reservations, as outlined above.}

Author Response 23 Aug 2016

Philip Schluter, University of Canterbury, Canterbury, New Zealand

Thank you greatly for your prompt, encouraging, and constructive feedback on our paper. Together with the comments made by the other two reviewers, your feedback has resulted in what we feel is a much improved paper. 
We would like to take this opportunity to make two notes. [1] The original submission was restricted to 1000 words in total - but, with permission from the journal, this constraint has been relaxed and we are able to include more material. This means we are able to broaden discussion of relevant issues and thus strengthen the manuscript. [2] This paper was submitted to the journal as an 'observation article', defined as: Observation Articles allow the description of a novel observation that may be unexpected, and possibly currently without explanation. An observation can be a phenomenon that has been identified in field work, in the laboratory or through experimental analysis (see: http://f1000research.com/forauthors/article-guidelines/observation-articles).

Title and Abstract: The title is catchy and appropriate given the content of the article.

Thank you for the positive comment.

Article content: The article is very well written and is easy to read and follow. The article would be much improved if it included an introduction to respondent drive sampling (RDS) with particular emphasis on the assumptions required for RDS to work (reaching equilibria and otherwise) written in clear terms to a lay audience. Describe what it means for the sampling process to be in equilibrium. Currently, readers do not have the necessary information to reach an informed conclusion as to why RDS did not work well in this instance or to assess whether it is applicable to their own work.

We thank the reviewer for describing our article as well written and easy to read and follow. We agree that an introduction to RDS would improve the article. Our original submission was limited by word restrictions and article scope, as outlined above. However, in this revision, we have included more details. Specifically, we have included text in the Introduction section (see second paragraph) providing a brief introduction to RDS, and describing equilibrium (see third paragraph). We have expanded the Results section (second paragraph) to describe why equilibrium was not reached in our study.

The rationale for RDS assumes the existence of a social network in which the probability of a tie between a wheelchair user and a randomly selected wheelchair user exceeds the probability of a tie between a wheelchair user and a nonuser wheelchair user. What evidence that this is true?

We had no evidence to suggest this was true or false and felt on balance that the use of RDS in our study was warranted as there was a reasonable enough chance that it could be true without evidence to the contrary. Conducting a revised search on PubMed, Google scholar, and Science Direct, we could find no literature explicitly addressing this issue (we have included a sentence in the first paragraph of the Discussion section describing this).

Do wheelchair users have sufficient social and other relationships importantly enough for them to be able to name sufficient other wheelchair users for the next round of sampling. Is it possible that many wheelchair users are isolates (in the network sense) because they are highly functional in everyday life and thus have reduced need for a community of wheelchair users? I don't know the answers to these questions and still don't as the needed background information is not provided in the article. 
This is indeed possible. The fact that a person uses a wheelchair does by no means infer that they know other people who use wheelchairs, let alone have strong internal networks with other wheelchair users. With respect to these questions we have included new text in the Discussion section (see first paragraph) regarding stigma and whether or not wheelchair users have sufficiently strong internal networks that enable random recruitment of other wheelchair users.

It would have been helpful to have been told the current state of knowledge about social networks among wheelchair users along with a connection of such knowledge to the assumptions under which RDS can yield unbiased population-level inferences. I would like to see some discussion of how RDS handles scenarios such as isolates in the wheelchair user network. Presumably, the only way such individuals make it into the sample is if they were randomly selected in the first (i.e., seed) wave.

As noted above, the current state of knowledge regarding the social networks among wheelchair users is unknown. As a result we cannot know if some wheelchair users are isolates. RDS theory assumes that the population being sampled comprises of a complete social network component, so in theory every person within their population has a probability of being sampled. In practice, to overcome isolated subpopulations, it is recommended that seeds from diverse subpopulations be selected. We have included a sentence to this effect in the second paragraph of the Discussion section.

Please add some general intuition for how RDS yields unbiased population-level estimates. Discuss the role of and derivation of individuals' sampling probabilities and thus sampling weights for computing population estimates.

Due to original word count, we had not included information clarifying that question two of our survey asked participants to provide an estimate of their network size (a requirement of RDS). Had our study produced in-equilibrium data (see new text in second paragraph in Introduction section, and new text in second paragraph of the Results section), sampling weights would have been allocated accordingly with those with smaller social network sizes being given a higher weight.

For example, the work of Thompson (2006a, 2006b, 2003, 2000 - see below - the 2012 text on Sampling and recent arXiv contributions) should be reviewed and used to inform the article.

References:

1. Thompson, Steven K. Targeted random walk designs. Survey Methodology 2006. 32:11-24.

2. Thompson, Steven K. Adaptive web sampling. Biometrics 2006. 62:1224-1234.

3. Chow, Mosuk, Thompson, Steven K. Estimation with link-tracing sampling designs - A Bayesian approach. Survey Methodology 2003. 29:197-205.

4. Thompson, Steven K., Frank, Ove. Model-based estimation with link-tracing sampling designs. Survey Methodology 2000. 26:87-98. 
Thank you greatly for suggesting these references, which were reviewed and indeed helped with our thinking. Because our study failed to produce in-equilibrium data that warranted further analysis and consideration of sampling weights and sampling probabilities, substantial engagement with the topics in these papers was felt to be beyond the scope of our paper.

Data (if applicable): Details about the design/data for the study are lacking. It is stated that 20 wheelchair users were the initial seeds of which 12 agreed to participate (60\% response rate). It then appears as though 4 of these 12 participants culled 7 further participants in the second stage of sampling. Question: Did the remaining 8 seeds not generate any wheelchair users (i.e., does the enrolment rate for the second stage equal 7/12) or was the plan to only ask $33 \%$ of the initial seeds for links to trace (in which case the enrolment rate is 7/4)?

All 12 seeds were asked to recruit 3 peers and for links to trace. Despite this, 8 seeds did not generate any more participants. The enrolment rate was $7 / 12$. To clarify recruitment/response rates, text has been included in the first paragraph of the Results section.

In the third stage, there was no addition to the sample-size. Were the 7 participants who entered at the second stage each asked to name other wheelchair users; is the enrolment rate at the third stage $0 / 7$ or $0 / x$, where $x<7$ ?

The enrolment rate was 0/7. All 7 participants were asked to recruit 3 peers and for links to trace, but no participants were recruited. To clarify recruitment/response rates, text has been included in the first paragraph of the Results section.

Was there any limit as to how many wheelchair users a seed to subsequently enrolled person could name? A lot of these questions would be answerable if the design of the RDS was described in the article!

Thank you for raising this issue. All seeds and subsequent participants were asked to recruit a maximum of 3 peers and for links to trace. Heckathorn (1997) recommends that each respondent is limited to 3 ID codes to pass on to others, to ensure that a broad array of participants is recruited, and to prevent the emergence of semi-professional recruiters. We have now included two sentences describing this in the second paragraph in the Methods section.

Conclusions: The discussion makes a number of good points that have appear sound and logical. The primary point made is that the current study may have failed because the wheelchair user population has different characteristics from populations where RDS has been used successfully. This is a valid point. But shouldn't this point have been considered when the study was designed?

Yes, a valid point. In retrospect the potential impact of this point could have attracted greater consideration. However, we had no evidence to suggest it would be detrimental to the success of our employment of RDS. We also acknowledged that our application of RDS with wheelchair users was unprecedented and novel, and many outcomes were simply unknown. In the absence of any reliable sampling frame or registry, we believed the 
potential success amongst this population (on balance) was greater than the risks.

Was there any prior information to inform the parameters of the RDS design? Maybe RDS would work quite well for the wheelchair user population as long as the number of initial seeds is much greater than 20. A more positively-framed Discussion might leave readers with a more balanced appreciation for using RDS in their own studies of wheelchair users or other new populations.

Since our employment of RDS, a number of points, that may have improved our experience, have been considered. These include selecting more diverse seeds, and meeting with seeds to provide more information to them. To summarise these points, new text has been included our revised manuscript (see second paragraph in Discussion section).

Competing Interests: No competing interests to disclose.

Reviewer Report 13 June 2016

\section{https://doi.org/10.5256/f1000research.9259.r14016}

(C) 2016 Smith J. This is an open access peer review report distributed under the terms of the Creative Commons Attribution License, which permits unrestricted use, distribution, and reproduction in any medium, provided the original work is properly cited.

John F. Smith

Postgraduate Tropical Medicine Program, Faculty of Medicine, Kohn Kaen University, Khon Kaen, Thailand

I support indexing this article as it as it appears to be a first-time test of the utility of Respondent Driven Sampling (RDS), a relatively novel sampling procedure, on a new "hidden" (hard to easily access) population--wheelchair riders. In this case it was not successful in recruiting sufficient respondents, but reporting that, and possible reasons for that outcome, could be useful for refining usage of the procedure.

I think the title and abstract are clear and provide good insight into the major points of the paper.

In addition to the possible reasons for low returns provided by the author I think it would be useful to include consideration of;

1. The typically low returns from internet surveys compared to face to face and pencil and paper surveys (see $1,2,3$ ). It is possible that promoting an internet survey was just insufficient to motivate the "seeds", or the second wave recruits, to recruit more members into the network, even with an iPad reward incentive.

2. Elaborating on the "stigmatization" issue raised in the discussion. The "mechanism by which perceived stigma may effect RDS sampling" rather than being unknown, may well be that other groups e.g., drug users, "men who have sex with men"(MSM), sex-workers etc as a result of stigma, may have stronger social networks and obvious gathering spaces and be 
easier to access via seeds. Seeds with these groups are often "leaders", advocates who are "out", support group leaders, health service liaison/volunteer workers who move easily within those communities, social gatherings, entertainment or work spaces. There are not the same social presses for wheelchair riders to have these group social connections or common activities.

3. Methodological point. The article focuses on RDS's value for accessing representative population samples for research seeking normative and epidemiological data and concomitant importance of avoiding/controlling sample selection bias. However, RDS could also be valuable for accessing qualitative data e.g., wheelchair riders' views/experiences with public policies/services etc where as wide a range of responses (variability) should be expected, indeed welcomed, rather than seen as requiring some statistical weighting or control procedure. Other forms of criterion for data "completeness" e.g., saturation could be used here. Another use for RDS is just to collect as large a sample as possible in hidden groups regardless of their representativeness. For example, RDS "seeds" have been used to recruit as many MSM as possible in "test and treat" outreach campaigns for blood testing for HIV and ante-retroviral treatment programs.

Overall a potentially useful addition to methodological literature on sampling procedures.

Competing Interests: No competing interests were disclosed.

\section{I confirm that I have read this submission and believe that I have an appropriate level of expertise to confirm that it is of an acceptable scientific standard, however I have significant reservations, as outlined above.}

Author Response 23 Aug 2016

Philip Schluter, University of Canterbury, Canterbury, New Zealand

Thank you greatly for your prompt, encouraging, and constructive feedback on our paper. Together with the comments made by the other two reviewers, your feedback has resulted in what we feel is a much improved paper.

We would like to take this opportunity to make two notes. [1] The original submission was restricted to 1000 words in total - but, with permission from the journal, this constraint has been relaxed and we are able to include more material. This means we are able to broaden discussion of relevant issues and thus strengthen the manuscript. [2] This paper was submitted to the journal as an 'observation article', defined as: Observation Articles allow the description of a novel observation that may be unexpected, and possibly currently without explanation. An observation can be a phenomenon that has been identified in field work, in the laboratory or through experimental analysis (see: http://f1000research.com/forauthors/article-guidelines/observation-articles).

I support indexing this article as it as it appears to be a first-time test of the utility of Respondent Driven Sampling (RDS), a relatively novel sampling procedure, on a new "hidden" (hard to easily access) population - wheelchair riders. In this case it was not successful in recruiting sufficient respondents, but reporting that, and possible reasons for that outcome, could be useful for 
refining usage of the procedure.

Thank you for recognising the novelty of this approach, together with your encouraging and supportive comments regarding our paper.

I think the title and abstract are clear and provide good insight into the major points of the paper

Thank you.

In addition to the possible reasons for low returns provided by the author I think it would be useful to include consideration of;

1. The typically low returns from internet surveys compared to face to face and pencil and paper surveys (see 1,2,3). It is possible that promoting an internet survey was just insufficient to motivate the "seeds", or the second wave recruits, to recruit more members into the network, even with an iPad reward incentive.

Yes, this is indeed a valid consideration. In response, we have included a sentence the second paragraph in our revised discussion specifically referring to how more informed and enthusiastic seeds could have encouraged greater response rates, acting as a potential mitigation strategy against the impersonal nature of electronic surveys.

1. Elaborating on the "stigmatization" issue raised in the discussion. The "mechanism by which perceived stigma may effect RDS sampling" rather than being unknown, may well be that other groups e.g., drug users, "men who have sex with men"(MSM), sex-workers etc as a result of stigma, may have stronger social networks and obvious gathering spaces and be easier to access via seeds. Seeds with these groups are often "leaders", advocates who are "out", support group leaders, health service liaison/volunteer workers who move easily within those communities, social gatherings, entertainment or work spaces. There are not the same social presses for wheelchair riders to have these group social connections or common activities.

Yes, this is valid and interesting point, and was also raised by another reviewer. In response, we have elaborated on the stigma issue by including new text in the first paragraph in the Discussion section addressing the area of perceived stigma, and whether or not wheelchair users have a sufficiently strong internal network that enable the random recruitment of other wheelchair users.

1. Methodological point. The article focuses on RDS's value for accessing representative population samples for research seeking normative and epidemiological data and concomitant importance of avoiding/controlling sample selection bias. However, RDS could also be valuable for accessing qualitative data e.g., wheelchair riders' views/experiences with public policies/services etc where as wide a range of responses (variability) should be expected, indeed welcomed, rather than seen as requiring some statistical weighting or control procedure. Other forms of criterion for data "completeness" e.g., saturation could be used here. Another use for RDS is just to collect as large a sample 
as possible in hidden groups regardless of their representativeness. For example, RDS "seeds" have been used to recruit as many MSM as possible in "test and treat" outreach campaigns for blood testing for HIV and ante-retroviral treatment programs.

The value of using the RDS in qualitative research is a very interesting concept, and could be raised in the Discussion. However, on balance we feel it may be beyond the scope of our paper here, which was to discuss our experience of unsuccessfully satisfying the requirements needed to produce a theoretically representative sample of the population with no sampling frame.

Overall a potentially useful addition to methodological literature on sampling procedures.

We appreciate these words and the additional references offered.

Competing Interests: No competing interests to disclose

Reviewer Report 09 May 2016

https://doi.org/10.5256/f1000research.9259.r13572

(C) 2016 Kokaua J. This is an open access peer review report distributed under the terms of the Creative Commons Attribution License, which permits unrestricted use, distribution, and reproduction in any medium, provided the original work is properly cited.

\section{Jesse Kokaua}

Pacific Islands Research \& Student Support Unit, University of Otago, Dunein, New Zealand

I think this paper has merit as a scientific publication, in that it adds to the body of research about RDS by its application to a "hidden" population, that by all accounts should be reasonable, but turned out to be not effective.

The abstract begins with a sentence that ends with a repeated point "rapid increasing life-span" and "accelerated general population ageing". One of those statements is redundant. Paragraph 2 of the introduction starts with international findings but does the second sentence beginning with "Despite this . ." refer to the NZ experience, or outside of the US and UK?

I feel that some points could be expanded upon:

The authors could expand, in the introduction, how the method improves the validity of, and reduces biases in, population-based estimates over other methods.

Examples of typical target populations for this method that are introduced in the discussion should move to the introduction, as it supports the novelty of this application.

I feel the results are two-fold. Firstly, the sample as described. I know its a moot point but I would 
be interested to know how big a sample is required to reach equilibrium.

Secondly, since this is a paper on the RDS, I would have liked to see a description of the total numbers of people approached in each wave, with eligible and ineligible participants. Thus reporting if the failure was a result of response, eligibility, or simply a lack of invitation to participate in subsequent waves.

This is an interesting application of a novel method of sampling from a group of people made hard-to-reach, it seems, as a result of their successful integration into contemporary New Zealand society. One characteristic of strongly stigmatised groups in New Zealand, such as those identified in the paper, are their tendency to establish strong internal networks, both informal and formal, that I feel would be useful to this type of methodology. It would be difficult for individuals with small networks to fulfill the random selection criteria for RDS. I take it that this may have influenced the less stigmatised wheelchair user population but it wasn't clearly stated if this was the case.

The paper reports a good example of where a useful method may not apply, in its initial format, to a particular interest group. However, it wasn't totally discounted as a potential approach but the reader is left without any suggestion as to how it might be modified to improve its take up, except for the suggestion of formative research, while fulfilling the assumptions required for RDS to provide unbiased population-wide estimates.

Competing Interests: No competing interests were disclosed.

\section{I confirm that I have read this submission and believe that I have an appropriate level of expertise to confirm that it is of an acceptable scientific standard, however I have significant reservations, as outlined above.}

Author Response 23 Aug 2016

Philip Schluter, University of Canterbury, Canterbury, New Zealand

Thank you greatly for your prompt, encouraging, and constructive feedback on our paper. Together with the comments made by the other two reviewers, your feedback has resulted in what we feel is a much improved paper.

We would like to take this opportunity to make two notes. [1] The original submission was restricted to 1000 words in total - but, with permission from the journal, this constraint has been relaxed and we are able to include more material. This means we are able to broaden discussion of relevant issues and thus strengthen the manuscript. [2] This paper was submitted to the journal as an 'observation article', defined as: Observation Articles allow the description of a novel observation that may be unexpected, and possibly currently without explanation. An observation can be a phenomenon that has been identified in field work, in the laboratory or through experimental analysis (see: http://f1000research.com/forauthors/article-guidelines/observation-articles).

I think this paper has merit as a scientific publication, in that it adds to the body of research about RDS by its application to a "hidden" population that by all accounts should be reasonable, 
but turned out to be not effective.

Thank you for your positive comments and summation. This, for us, was the basis for opting for the "novel observation" article type.

The abstract begins with a sentence that ends with a repeated point "rapid increasing life-span" and "accelerated general population ageing". One of those statements is redundant.

Although the intention was to make two important points, the first referring to wheelchair users and the second referring to the general population, we agree that this is unnecessarily confusing. We have revised this sentence to: "Internationally, wheelchair users are an emerging demographic phenomenon, due to their rapidly increasing lifespan."

Paragraph 2 of the introduction starts with international findings but does the second sentence beginning with "Despite this.. " refer to the NZ experience, or outside of the US and UK?

This appears to be an international trend. However, to be clearer we have included "in New Zealand and Australia" and inserted an apposite reference.

I feel that some points could be expanded upon: The authors could expand, in the introduction, how the method improves the validity of, and reduces biases in, population-based estimates over other methods.

Agreed - indeed, a similar point was raised by another reviewer. Our original submission was limited in scope due to the word restrictions, as outlined above. However, in this revision, we have included more information and discussion. Specifically, we have included text providing a brief introduction to RDS (See second paragraph in Introduction section).

Examples of typical target populations for this method that are introduced in the discussion should move to the introduction, as it supports the novelty of this application.

Thank you for this comment; it indeed strengthens our introduction. In our revised manuscript we have included the following sentence with paragraph one of the Introduction: "RDS has traditionally been used to sample 'hidden' populations with inadequate sampling frames, such as those with greater risk of HIV, including injecting drug users."

I feel the results are two-fold. Firstly, the sample as described. I know it's a moot point but I would be interested to know how big a sample is required to reach equilibrium.

We also felt that this information would strengthen our paper, but omitted it initially - due to the word count restrictions. In this version, within the Introduction, we now explicitly add an explanation about the concept of equilibrium and how it is reached (see third paragraph), while the second paragraph in the Results section explains specifically why our sample failed to satisfy the threshold for in-equilibrium data. 
Secondly, since this is a paper on the RDS, I would have liked to see a description of the total numbers of people approached in each wave, with eligible and ineligible participants. Thus reporting if the failure was a result of response, eligibility, or simply a lack of invitation to participate in subsequent waves.

These details would be hard, if not ethically impossible, to determine. Consistent with RDS protocol, an email was sent to people asking them to send on the invitation to three people, but there was no obligation for us (the researchers) to receive confirmation about whether all three recruitment codes were successfully allocated and distributed. Nevertheless, more information has been included in the Methods section (second paragraph) regarding how participants were asked to recruit three others, and the first paragraph in the Results section, which clarifies recruitment and response rates.

This is an interesting application of a novel method of sampling from a group of people made hard-to-reach, it seems, as a result of their successful integration into contemporary New Zealand society. One characteristic of strongly stigmatised groups in New Zealand, such as those identified in the paper, are their tendency to establish strong internal networks, both informal and formal, that I feel would be useful to this type of methodology. It would be difficult for individuals with small networks to fulfil the random selection criteria for RDS. I take it that this may have influenced the less stigmatised wheelchair user population but it wasn't clearly stated if this was the case.

Yes, this is valid and interesting point and was also raised by another reviewer. In response, we have included two sentences in the first paragraph of the Discussion section addressing the area of perceived stigma and whether or not wheelchair users have sufficiently strong internal networks that enable the random recruitment of other wheelchair users.

The paper reports a good example of where a useful method may not apply, in its initial format, to a particular interest group. However, it wasn't totally discounted as a potential approach but the reader is left without any suggestion as to how it might be modified to improve its take up, except for the suggestion of formative research, while fulfilling the assumptions required for RDS to provide unbiased population-wide estimates.

Thank you for this comment that will strengthen our paper. In response we have included more information in the second paragraph of the Discussion section about potential ways to improve similar studies, namely, selecting diverse seeds with larger networks and providing greater information and education to those seeds.

Competing Interests: No competing interests to disclose 
The benefits of publishing with F1000Research:

- Your article is published within days, with no editorial bias

- You can publish traditional articles, null/negative results, case reports, data notes and more

- The peer review process is transparent and collaborative

- Your article is indexed in PubMed after passing peer review

- Dedicated customer support at every stage

For pre-submission enquiries, contact research@f1000.com 